\title{
Irradiation des personnes par les tubes cathodiques de télévision
}

\author{
G. WOLBER* \\ (Manuscrit reçu le 3 décembre 1983)
}

\begin{abstract}
RÉSUMÉ
L'extension du marché des récepteurs de télévision en couleur durant la dernière décennie et l'emploi de plus en plus fréquent des consoles de visualisation polychromes en informatique ont accru la population exposée aux rayons $X$ émis par les tubes cathodiques. Dans le même temps, les perfectionnements techniques de ces tubes ont diminué l'irradiation individuelle des utilisateurs dans un rapport d'au moins 20. En s'appuyant sur des calculs et des résultats expérimentaux, ce rapport fait le point sur les niveaux d'irradiation possibles actuellement au voisinage des écrans des récepteurs et des consoles de visualisation. Seuls, les tubes trichromes sont susceptibles d'emettre des rayonnements détectables et les doses délivrées ne dépassent pas $1 \mu \mathrm{Sv}(0,1 \mathrm{mrem})$ par an.
\end{abstract}

\section{ABSTRACT}

The great extent of the sale of colour TV receivers during the last decade and the more and more frequent use of trichromatic video display units in data processing have increased the population exposed to X-rays emitted by cathode-ray tubes. In the same time, because of technical improvements made on these tubes, the individual exposure of users has decreased by a ratio over 20. Based on numerical calculations and experimental results, this report gives a review on exposure levels presently possible near TV screens and video display units. Only three-colour tubes may emit detectable radiations and the delivered doses do not exceed $1 \mu$ Sv per year.

On assiste, depuis une dizaine d'années, à une extension très rapide du marché des récepteurs de télévision en couleurs facilitée, d'une part, par la généralisation des programmes en couleur et, d'autre part, par des progrès technologiques qui ont abaissé les coûts de fabrication et de maintenance des appareils en améliorant leur fiabilité.

Du côté professionnel, l'emploi des moyens informatiques par l'intermédiaire de terminaux à écran cathodique se développe à grande échelle. On tend de plus en plus à utiliser des écrans polychromes qui amènent de plus grandes possibilités d'emploi : conception assistée par

* Electricité de France, Direction Production - Transport, Comité de Radioprotection, 39, rue de Washington, 75384 Paris Cedex 08. 


\section{G. WOLBER}

ordinateur, analyse de données, traitement d'images, réalisation de schémas, exploitation de processus industriels.

Tous ces moyens utilisent les techniques de télévision ou apparentées à celle-ci, et sont des sources potentielles de rayons $\mathrm{X}$ à cause des tensions élevées mises en jeu. En ce qui concerne la sécurité des utilisateurs, ces matériels doivent être conformes à la norme AFNOR C 92-130 [1]. Dans le domaine de l'irradiation par les rayons $X$, les limites admissibles ont toujours été largement respectées et on estimait, en 1970, la dose moyenne reçue par les téléspectateurs à $20 \mu \mathrm{Sv} / \mathrm{an}$ (2 mrem/an). L'évolution de la technologie des équipements de télévision a simplifié les schémas et diminué le rayonnement $X$ dans des rapports pouvant dépasser 100 au point que les niveaux ne peuvent plus être mesurables par les appareils classiques de dosimétrie. Nous allons voir pourquoi dans ce qui suit.

\section{EVOLUTION DU RAYONNEMENT DES TUBES CATHODIQUES DE TELEVISION}

\subsection{Principe du tube cathodique}

Le tube cathodique réalise la synthèse des images. II est constitué d'une ampoule en verre vide d'air pour laquelle on peut décrire trois parties (fig. 1). Un corps cylindrique à l'arrière, appelé col, contient un canon à électrons destiné à engendrer un faisceau étroit d'électrons accélérés sous des tensions de l'ordre de la dizaine de kilovolts. La face avant en verre épais, la dalle, est recouverte sur sa surface intérieure d'une couche fluorescente, le luminophore ${ }^{(1)}$ : c'est elle qui constitue l'écran proprement

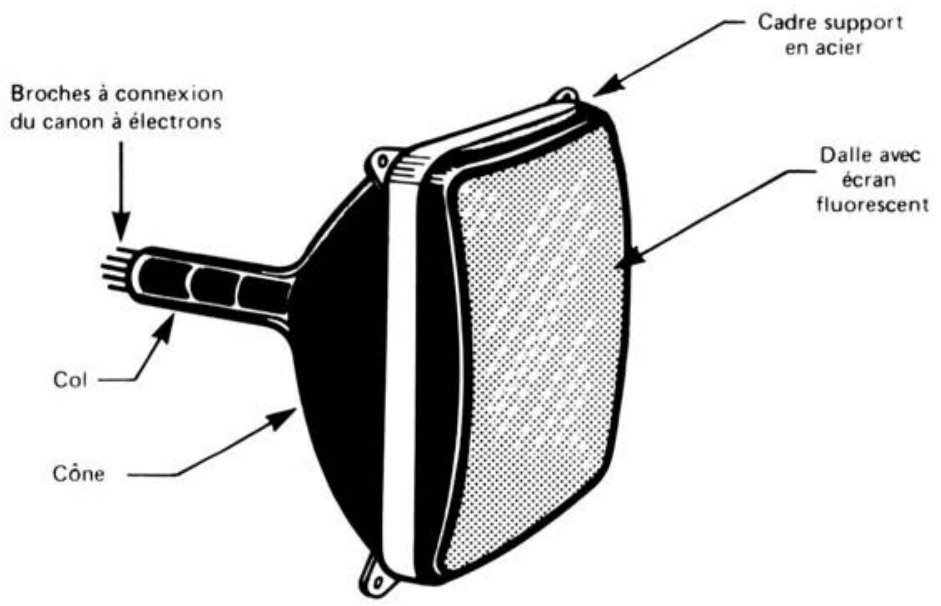

Fig. 1. - Vue extérieure d'un tube cathodique de télévision.

(1) Terme choisi pour la traduction du mot anglais " phosphor" afin de le distinguer de l'espèce chimique phosphore. 
dit. Enfin, le cône, qui réunit le col et la dalle, porte l'électrode de postaccélération du faisceau électronique. Cette électrode est formée par un dépôt conducteur sur la surface intérieure du cône et est reliée à l'alimentation haute-tension par la "corne" d'anode, connexion qui traverse la partie supérieure du cône.

Le faisceau d'électrons est focalisé et dirigé en un point choisi de l'écran par un système de déviation magnétique ou de balayage entourant le col près de sa jonction avec le cône. La couche de luminophore s'illumine sous l'impact des électrons en formant un point lumineux appelé spot qui se déplace sur l'écran. Pour engendrer une image, la brillance du spot suit les variations du courant du faisceau d'électrons produites par la chaîne d'amplification image ou vidéo de l'équipement. Cette chaîne fournit également les signaux de synchronisation du balayage nécessaires pour positionner l'image.

Tout ce qui précède concerne les tubes monochromes. Les tubes d'affichage graphique à pénétration procèdent de techniques voisines : l'écran est revêtu de deux couches superposées de luminophores et les électrons atteignent une ou deux couches suivant leur tension d'accélération échelonnée entre 10 et $18 \mathrm{kV}$. On arrive ainsi à reproduire des courbes et des textes de grande finesse dans les gammes de couleur du rouge au vert en passant par l'orangé et le jaune. Certaines combinaisons de luminophores permettent d'aller jusqu'au blanc.

Les tubes polychromes forment les images colorées par juxtaposition de trois images dans les couleurs élémentaires : rouge, vert et bleu. Le système de reproduction d'images est triplé : chaque faisceau d'électrons ne peut atteindre que le luminophore correspondant à sa couleur car il traverse un masque perforé destiné à assurer la séparation des trois images. Pour obtenir des performances comparables aux systèmes monochromes, il faut des tensions d'accélération plus élevées, 25 à $30 \mathrm{kV}$ au lieu de 10 à $12 \mathrm{kV}$, et des courants plus importants, $2 \mathrm{~mA}$ au lieu de 0,5 mA, pour une image blanche uniforme à la brillance nominale.

\subsection{Evolution du rayonnement $X$ parasite}

Les électrons, du fait qu'ils sont accélérés à des tensions de 10 à $30 \mathrm{kV}$, engendrent des rayons $X$ mous de freinage. Avec des intensités de faisceau de l'ordre du milliampère, les alimentations haute tension mettent en jeu des puissances de quelques dizaines de watts. Les tubes cathodiques sont donc des sources potentielles de rayons $X$ et ceux-ci ont posé des problèmes pour la radioprotection des téléspectateurs au début de la télévision en noir et blanc, puis de nouveau aux débuts de la télévision en couleurs aux Etats-Unis [3]. A la suite d'un certain nombre de perfectionnements de la technologie des tubes cathodiques et de leurs systèmes de commande et d'alimentation, le niveau d'irradiation est descendu très bas.

Dans les années 1960 , on a cherché à protéger les utilisateurs des conséquences de l'implosion des tubes en doublant la dalle d'un verre épais d'1 cm (tube "twin panel"), puis on a épaissi cette dalle en lui donnant un profil de voûte ; en outre, l'écran a été enserré dans un cadre 
d'acier servant de fixation au bâti de l'équipement (tube auto-protégé). Ensuite, vers 1968, le rendement des luminophores a été amélioré : s'ils sont toujours à base de sulfure de zinc pour les tubes monochromes, ils utilisent des sels complexes dopés aux terres rares pour le rouge dans les tubes polychromes. La réponse en a été améliorée, simplifiant les circuits vidéo. A la même époque, les ampoules ont été fabriquées en verre chargé aux métaux lourds et on a revu la conception des tubes haute-tension des circuits d'alimentation : le niveau de rayonnement $X$ était alors descendu au moins d'un facteur 10. Enfin, l'usage généralisé des semiconducteurs dans les équipements, depuis 1972, a supprimé définitivement les sources de rayons $X$ extérieures au tube cathodique.

En quinze ans, le niveau d'irradiation par les rayons $X$ parasites a donc été réduit dans un rapport d'au moins 50 .

\section{BUT ET HYPOTHESES DU CALCUL}

Le calcul théorique de la distribution spatiale et spectrale du rayonnement $X$ à partir des caractéristiques du faisceau électronique est complexe et dépasse les objectifs visés par cet article. D'autre part, il n'est plus possible de donner des résultats de mesure de ce rayonnement à la distance de vision, son niveau étant en-dessous du seuil de mesure, mais on peut encore relever des valeurs au voisinage de l'écran avec des techniques de laboratoire. La démarche à suivre est de calculer les variations relatives de l'intensité du rayonnement dans l'espace pour en déduire ensuite des estimations des niveaux d'irradiation des utilisateurs moyennant quelques hypothèses simplificatrices.

Le rayonnement de freinage engendré par le faisceau d'électrons n'est pas isotrope : son intensité varie avec l'angle que font les rayons émis avec l'axe du faisceau. Ici, le rapport entre le maximum et le minimum est de l'ordre de 2 . Comme les rayons obliques par rapport à la surface de la dalle sont fortement absorbés, tout se passe comme si le rayonnement à l'origine était isotrope, c'est-à-dire indépendant de la direction d'émission.

Dans l'état actuel de la technique, les tubes monochromes ne produisent pas de rayonnements $X$ mesurables. Le spectre de freinage d'électrons à $12 \mathrm{kV}$ s'échelonne entre 2 et $11 \mathrm{keV}$. Avec une ampoule en verre à la chaux de $8 \mathrm{~mm}$ d'épaisseur, un faisceau de photons de $11 \mathrm{keV}$ est atténué dans un rapport de $10^{21(1)}$. En d'autres termes, un faisceau de photons de $11 \mathrm{keV}$ entraînant un débit de dose de $10 \mathrm{~Gy} / \mathrm{h}(1000 \mathrm{rad} / \mathrm{h})$ donnerait, à la sortie, un débit de dose de $10^{-20} \mathrm{~Gy} / \mathrm{h}\left(10^{-18} \mathrm{rad} / \mathrm{h}\right)$. Pour donner une idée de la faiblesse de cette valeur, il suffit de se rendre compte qu'une exposition annuelle de $1000 \mathrm{~h}$ à ce débit de dose entraîne une dose accumulée de $10^{-17}$ Gy $\left(10^{-15} \mathrm{rad}\right)$ par an.

(1) Pour un verre à la chaux, le cœfficient d'atténuation linéique $\mu$ est de l'ordre de $60 \mathrm{~cm}^{-1}$ à $11 \mathrm{keV}$. Le facteur d'atténuation entrée-sortie du faisceau traversant $8 \mathrm{~mm}$ de ce verre est $\mathrm{e}^{\mu \mathrm{e}}=\mathrm{e}^{(60 \times 0,8)}=\mathrm{e}^{48} \simeq 10^{21}$. 
Pour les tubes polychromes où la tension d'accélération nominale est de $25 \mathrm{kV}$, le spectre de freinage se situe dans la bande d'énergie de 16 à $22 \mathrm{keV}$. Le même verre à la chaux atténue un faisceau de photons de $20 \mathrm{keV}$ dans un rapport 600(1). Avec un verre au plomb, le facteur d'atténuation est relevé à 160000 soit environ 300 fois plus ${ }^{(1)}$. La différence d'ordre de grandeur de ces facteurs montre que seuls les tubes polychromes sont susceptibles de présenter des niveaux de rayonnement $\mathrm{X}$ mesurables.

Afin d'évaluer l'importance maximale possible de l'irradiation, les calculs concerneront un tube cathodique polychrome de grande taille (diagonale $47 \mathrm{~cm}$ ) et de tension d'accélération élevée $(25 \mathrm{kV})$.

\section{CALCuls efFectues}

\subsection{Spectre étudié}

Comme on l'a indiqué précédemment, le spectre de rayons $X$ émergeant de la paroi du tube cathodique se situe dans une bande centrée autour de $20 \mathrm{keV}$. D'après les mesures effectuées pour des récepteurs grand public [15] et des calculs théoriques, il a l'allure de la figure 2. Pour les calculs dosimétriques, on fait une bonne approximation en supposant que le faisceau émergent est composé de photons monoénergétiques de $20 \mathrm{keV}$.

A ces énergies, l'interaction des photons avec la matière est essentiellement un effet photoélectrique. La diffusion de Rayleigh, identique à celle des photons lumineux dans les gaz, entre pour quelques pour cent dans l'atténuation du faisceau et l'effet Compton est négligeable. Les électrons secondaires ont un libre parcours maximal de $3,2 \mathrm{~cm}$ dans l'air et $7 \mu \mathrm{m}$ dans l'eau : dans tous les points qui nous intéressent, l'équilibre électronique est atteint. II n'y a donc pas d'effet de build-up (2) et on a le droit d'assimiler l'atténuation des photons du faisceau incident à celle du débit de dose.

\subsection{Méthodes de calcul}

Les calculs se rapportent principalement aux écrans rectangulaires, les plus répandus. A titre de comparaison, certains points de calcul ont été vérifiés pour des écrans circulaires et ont montré que, tous paramètres égaux par ailleurs, le débit de dose ne dépend que de la surface éclairée et du rayon de courbure de l'écran.

(1) Avec les photons de $20 \mathrm{keV}$, le cœfficient d'atténuation linéique $\mu$ est de l'ordre de $8 \mathrm{~cm}^{-1}$ pour les verres à la chaux et $15 \mathrm{~cm}^{-1}$ pour les verres au plomb. Dans ces conditions, la traversée de $8 \mathrm{~mm}$ de verre entraîne une atténuation d'un facteur $\mathrm{e}^{6,4} \simeq 600$ pour le verre à la chaux et $\mathrm{e}^{12} \simeq 160000$ pour le verre au plomb.

(2) L'effet de build-up est une conséquence de la diffusion des photons par l'effet Compton: l'atténuation de la dose, quand le faisceau pénètre dans la matière, est inférieure à l'atténuation des photons incidents. 


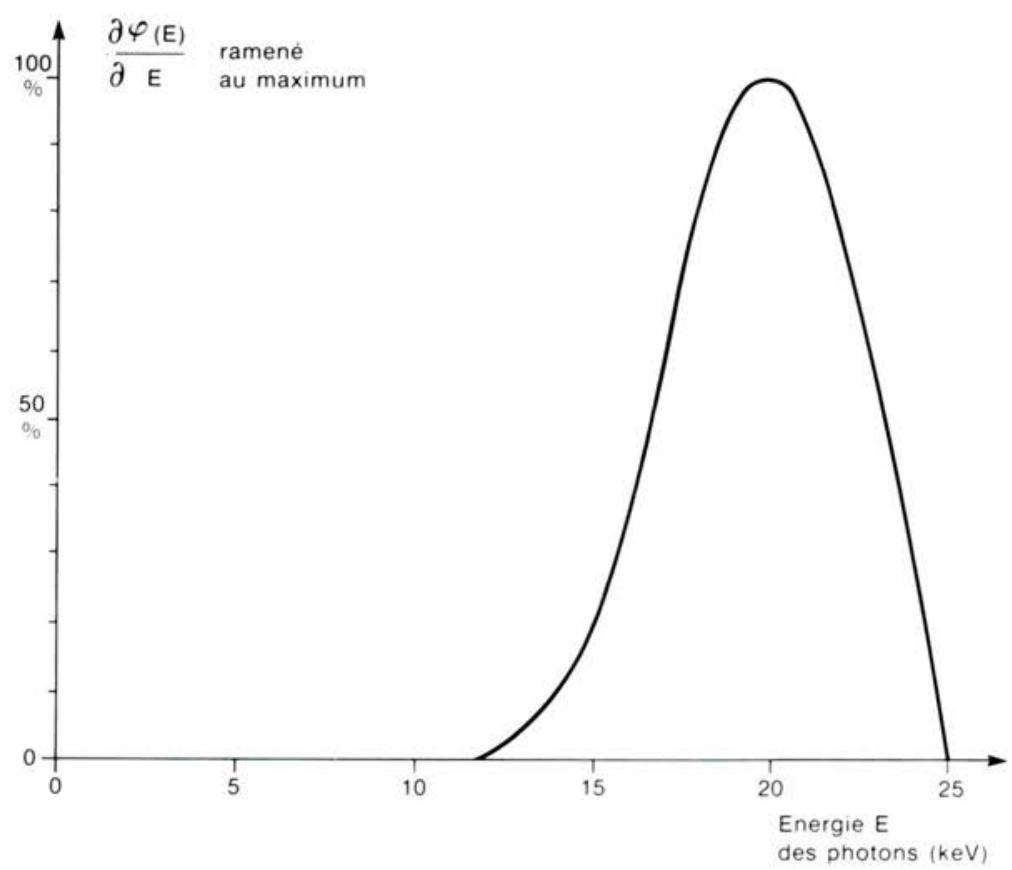

Fig. 2. - Spectre d'émission $X$ en sortie de l'écran de verre du tube cathodique. D'après Strackee et Van Dongen [15].

Les rayonnements latéraux sont entièrement absorbés par les blindages magnétiques placés autour du cône. Les rayons $X$ émis vers l'avant par le spot sont rapidement absorbés quand l'incidence est de plus en plus oblique. Ainsi, pour le matériel étudié ici, les rayons $X$ émergent de la dalle à l'intérieur d'un cône de $30^{\circ}$ de demi-angle d'ouverture. Les variations d'intensité de ce rayonnement sont essentiellement le résultat de l'atténuation du faisceau à travers le verre et l'hypothèse de l'isotropie est absolument acceptable.

Le tube étudié est du type autoprotégé dont l'ampoule est en verre à fort cœfficient d'absorption ( $\mu=15 \mathrm{~cm}^{-1}$ à $\left.20 \mathrm{keV}\right)$ d'une épaisseur moyenne de $8 \mathrm{~mm}$. Pour des tubes de plus grandes dimensions, les épaisseurs de verre sont peu différentes et l'augmentation du rayonnement due à l'accroissement de surface d'écran est compensée par l'effet de la distance d'observation plus élevée pour avoir le même confort visuel.

Pour effectuer les calculs qui suivent, les formules employées sont tirées de Fitzgerald, Brownell et Mahoney, 1976 [10] et les données proviennent d'Attix et Roesch [2], de R. Schaeffer [9] et des documents 
des constructeurs. Les résultats ont été obtenus par intégration numérique.

\subsection{Intensité du rayonnement au voisinage de l'écran : incidence sur l'interprétation des mesures}

Les cartes isodoses à $5 \mathrm{~cm}$ de la surface de la dalle sont semblables à celles de la figure 3 . Ces mêmes cartes à la distance de $10 \mathrm{~cm}$ et plus montrent que, pour un écran plan, le débit de dose ne varie pas de plus de $1 \%$ si cet écran est vu du point de mesure sous un angle solide supérieur à celui d'un cône de $45^{\circ}$ de demi-angle d'ouverture. Les écrans à surface sphérique, les plus courants, donnent des cartes isodoses semblables à celles des écrans plans si l'on se place sur une surface parallèle à la dalle. Dans ce cas, le débit de dose varie comme l'inverse de la distance de cette surface au centre de la sphère. Pour un écran de $47 \mathrm{~cm}$ de diagonale, le rayon de cette sphère est de l'ordre de $70 \mathrm{~cm}$ et la dé-

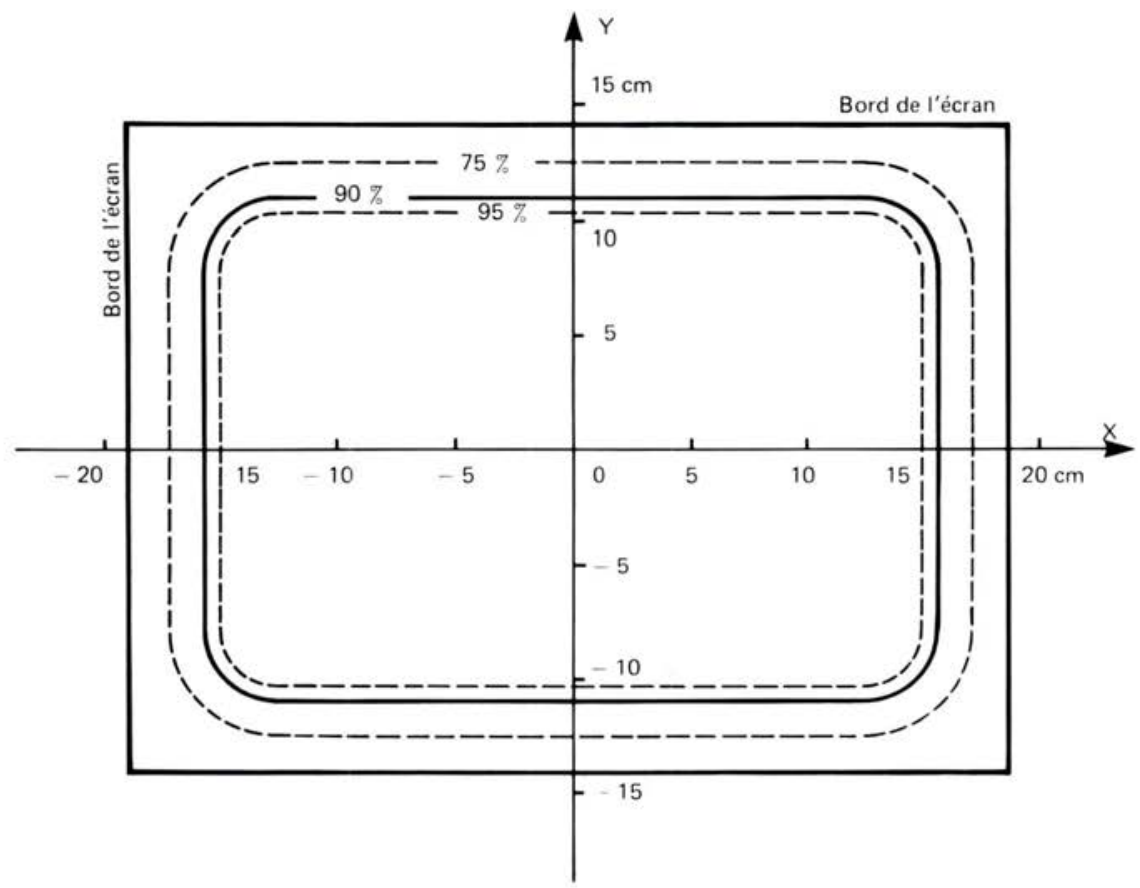

Fig. 3. - Courbes isodoses pour des points situés à $5 \mathrm{~cm}$ de la paroi đu tube cathodique côté écran. Valeurs normalisées à celle relative au point situé sur l'axe du tube. Ecran rectangulaire de $47 \mathrm{~cm}$ de diagonale. 
croissance du débit de dose, entre le contact de la dalle et la distance de $10 \mathrm{~cm}$, est limitée à $30 \%$. On peut donc, sans perdre notablement de sensibilité, placer un dosimètre à une distance de la dalle qui peut atteindre $10 \mathrm{~cm}$ : cela évite d'être perturbé par les charges électriques provenant des courants de fuite à travers le verre de l'ampoule. Les propriétés précédentes sont vraies si la dalle a une épaisseur constante. Les tolérances de fabrication peuvent introduire des fluctuations non négligeables dans le débit de dose mesuré à distance constante de la dalle : une tolérance de $0,5 \mathrm{~mm}$ sur une épaisseur de verre de $8 \mathrm{~mm}$ peut entraîner des variations du niveau d'irradiation de 5 \%.

\subsection{Diagramme de rayonnement dans l'espace}

Le spectre du rayonnement venant du tube cathodique se situe dans une bande étroite et ne dépend pas de la direction d'émission : cette bande est comprise entre 16 et $25 \mathrm{keV}$ avec un maximum autour de $20 \mathrm{keV}$.

La variation du débit de dose le long de l'axe du tube est représentée par la figure 4 pour des distances à la surface de l'ampoule de 0 à 2,50 m. On retrouve les résultats théoriques et expérimentaux de $\mathrm{L}$. Strackee et R. Van Dongen [15] et ceux de M. Berthaud et H. Penotet [3]. Les valeurs sont rapportées à celles mesurées à $5 \mathrm{~cm}$ de la surface du tube.

Conformément aux prévisions, on retrouve, pour l'écran plan, la constance de l'intensité du rayonnement de la surface du tube jusqu'à une distance de $20 \mathrm{~cm}$, soit environ la demi-diagonale de l'écran. Ceci s'explique aisément car l'atténuation du rayonnement est essentiellement due au verre et les rayons obliques sont très rapidement absorbés. Le rayonnement d'un point de l'écran fluorescent ne traverse l'ampoule que s'il se trouve dans un cône de demi-angle d'ouverture de 30 à $45^{\circ}$. Le point de mesure ne voit donc que la partie de l'écran comprise dans ce cône et l'effet de distance est compensé par l'accroissement de surface d'écran vu de ce point. La loi de variation de $1 / d^{2}$ des sources ponctuelles ne s'observe qu'au-delà de $75 \mathrm{~cm}$ où le niveau est tombé à $20 \%$ de la valeur initiale. Avec les écrans courbes, le niveau de rayonnement par rapport à sa valeur mesurée à $5 \mathrm{~cm}$ de distance, varie de $+15 \%$ au contact de la dalle à $-40 \%$ à la distance de $25 \mathrm{~cm}$.

En étudiant les variations d'intensité du rayonnement hors de l'axe du tube on constate que le diagramme de rayonnement, à surface éclairée égale, diffère très peu de celui d'un écran circulaire et est donc pratiquement de révolution autour de l'axe du tube. Un de ces diagrammes est donné à la figure 5 pour notre écran courbe de $47 \mathrm{~cm}$ de diagonale. On voit que l'essentiel du rayonnement est contenu dans un tronc de cône de demi-angle d'ouverture $30^{\circ}$ dans le cas de l'écran plan de même surface. On verra plus loin que, finalement, c'est le visage et la partie haute du tronc qui reçoivent la majeure partie du rayonnement.

\section{CALCUL DES DOSES DE RAYONNEMENT}

\subsection{Conditions d'irradiation}

Deux cas typiques peuvent être envisagés: le téléspectateur qui regarde en moyenne son récepteur couleur $2 \mathrm{~h}$ par jour à une distance 


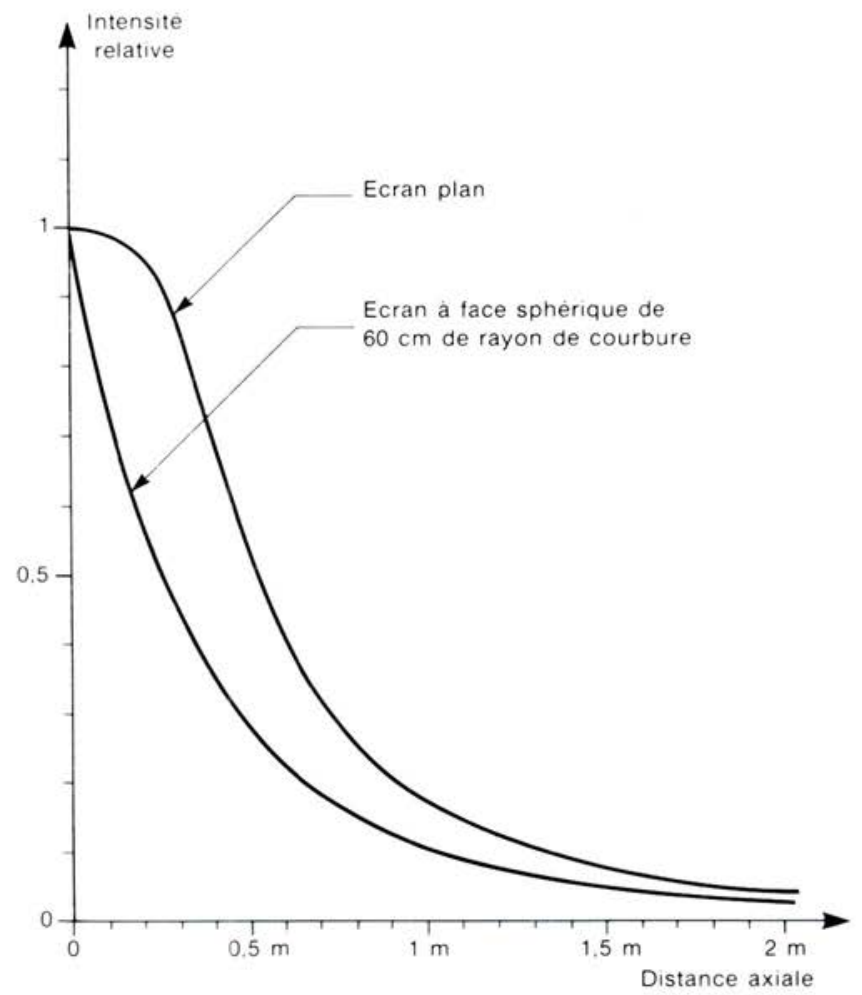

Fig. 4. - Variation du niveau de rayonnement le long de l'axe du tube. Les valeurs sont rapportées à celle donnée par l'écran plan à $5 \mathrm{~cm}$ de cet écran.

Variations comparées pour un écran rectangulaire plan de $47 \mathrm{~cm}$ de diagonale et de ce même écran à face sphérique de $70 \mathrm{~cm}$ de rayon de courbure.

Tension d'accélération de $25 \mathrm{kV}$. Verre de $8 \mathrm{~mm}$ d'épaisseur avec cœfficient d'atténuation $\mu=15 \mathrm{~cm}^{-1}$.

de $2 \mathrm{~m}$ et le travailleur sur console de visualisation couleur regardant l'écran à $60 \mathrm{~cm}$ de distance pendant $1400 \mathrm{~h}$ par an.

\subsection{Téléspectateur à $2 \mathrm{~m}$ de l'écran}

Tous les écrans de télévision à vision directe sont à face courbe. L'axe de l'écran du récepteur est horizontal et, dans le cas le plus défavorable, 
le récepteur est disposé assez près du sol. Le téléspectateur a des positions variables par rapport à cet écran mais on considère qu'en moyenne il est installé dans un fauteuil et regarde dans l'axe de l'écran. II se trouve incliné par rapport à cet axe comme l'indique la figure 5 . Le diagramme isodose indique que le champ d'irradiation varie peu entre la tête et l'abdomen du sujet et qu'on a une estimation majorante de la dose à $30 \%$ en supposant cette dose constante.

En reprenant les méthodes de calcul de R. Schaeffer [9] qui tiennent compte de la transmission air-eau équivalente à la transition air-tissus mous de l'homme, on obtient les facteurs de conversion entre le débit de dose mesuré à $5 \mathrm{~cm}$ de la surface de l'écran et les organes du sujet exposé (tableau I).

Les données anatomiques sont celles de l'homme de référence de la Commission internationale de protection radiologique (CIPR) [5].

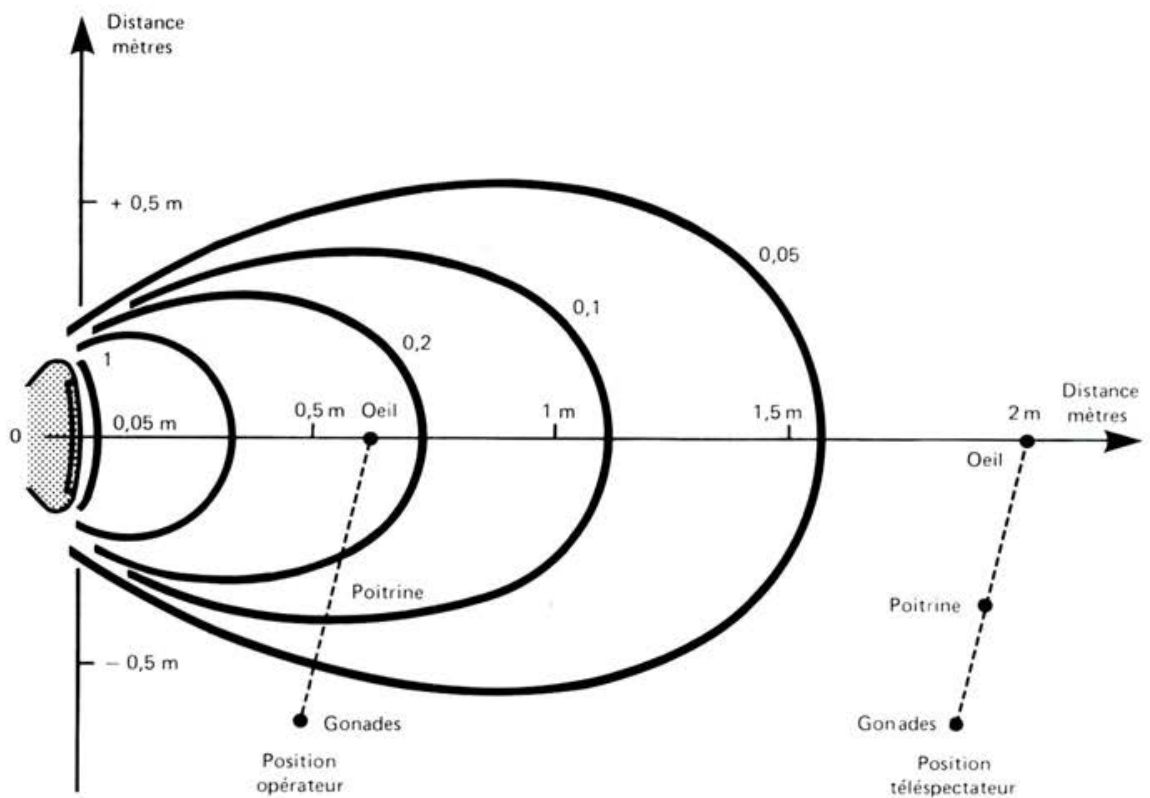

Fig. 5. - Courbes isodoses dans le plan vertical pour un tube polychrome de $47 \mathrm{~cm}$ de diagonale à face sphérique de $70 \mathrm{~cm}$ de rayon et alimenté à $25 \mathrm{kV}$. Valeurs normalisées à celle sur l'axe à $5 \mathrm{~cm}$ du tube. 


\section{TABLEAU I}

Facteurs de conversion calculés pour obtenir la dose aux organes critiques d'un téléspectateur regardant son récepteur à une distance de $2 \mathrm{~m}$.

\begin{tabular}{|c|c|c|c|}
\hline $\begin{array}{l}\text { Organes soumis } \\
\text { au risque } \\
\text { d'irradiation }\end{array}$ & $\begin{array}{l}\text { Rapport de la dose } \\
\text { à la surface du point } \\
\text { de pénétration dans } \\
\text { le corps à la dose } \\
\text { à } 5 \mathrm{~cm} \text { de l'écran }\end{array}$ & $\begin{array}{c}\text { Rapport de la dose } \\
\text { à l'organe } \\
\text { à la dose à la surface } \\
\text { du corps }\end{array}$ & $\begin{array}{l}\text { Rapport de la dose } \\
\text { à l'organe à la dose } \\
\text { à } 5 \mathrm{~cm} \text { de l'écran }\end{array}$ \\
\hline Oeil (cristallin) & \multirow{6}{*}{$3,5.10^{-2}$} & 0,81 & $2,84 \cdot 10^{-2}$ \\
\hline Crâne (os) & & 0,77 & $2,69.10^{-2}$ \\
\hline $\begin{array}{l}\text { Mœlle osseuse } \\
\text { (sternum) }\end{array}$ & & 0,56 & $1,96 \cdot 10^{-2}$ \\
\hline Sein (femme) & & 0,68 & $2,38.10^{-2}$ \\
\hline Testicules & & 0,56 & $1,96.10^{-2}$ \\
\hline Ovaires & & 0,02 & $7.10^{-4}$ \\
\hline
\end{tabular}

Ces résultats nous permettent de calculer les équivalents de dose annuels reçus par les téléspectateurs et d'observer leur évolution au cours des quinze dernières années (tableau II). La première série de valeurs correspond au débit d'équivalent de dose limite de $1 \mu \mathrm{Sv} / \mathrm{h}(0,1 \mathrm{mrem} / \mathrm{h})$, mesuré à $5 \mathrm{~cm}$ de l'écran, que les constructeurs s'étaient fixés avant 1972 [3]. Actuellement, la moyenne des mesures se situe autour de $0,2 \mu \mathrm{Sv} / \mathrm{h}$ (20 $\mu \mathrm{rem} / \mathrm{h})$, ce qui aboutit à la deuxième série de valeurs.

\section{TABLEAU II}

Equivalents de dose annuels reçus par les téléspectateurs avant et après 1972.

\begin{tabular}{|l|c|c|}
\hline \multirow{2}{*}{$\begin{array}{c}\text { Organes soumis } \\
\text { au risque d'irradiation }\end{array}$} & \multicolumn{2}{c|}{ Equivalents de dose annuels (mSv) } \\
\cline { 2 - 3 } & Avant 1972 & Après 1972 \\
\hline Cristallin & 0,021 & 0,0042 \\
Crâne & 0,020 & 0,0040 \\
Mœlle osseuse & 0,014 & 0,0028 \\
Sein & 0,017 & 0,0034 \\
Testicules & 0,014 & 0,0028 \\
Ovaires & $5.10^{-4}$ & $10^{-4}$ \\
\hline
\end{tabular}

En comparant ces valeurs avec les limites admissibles pour les personnes du public données par le tableau III, on constate qu'avant 1972, les organes critiques étaient irradiés au maximum à $3 / 100^{e}$ de ces limites.

Si l'on fait le calcul des équivalents de dose effectifs de la CIPR [6] séparément pour les hommes et les femmes selon les données de Kramer et Drexler [11], on constate que les résultats s'écartent peu de 0,015 mSv 
TABLEAU III

Limites annuelles d'équivalents de dose pour les personnes du public.

\begin{tabular}{|l|c|}
\hline $\begin{array}{c}\text { Organes soumis } \\
\text { au risque } \\
\text { d'irradiation }\end{array}$ & $\begin{array}{c}\text { Limite annuelle } \\
\text { d'équivalent de dose } \\
\text { (mSv/an) }\end{array}$ \\
\hline Cristallin & 15 \\
Crâne (os) & 30 \\
Mœlle osseuse & 5 \\
Sein & 15 \\
Testicules & 5 \\
Ovaires & 5 \\
\hline
\end{tabular}

(1,5 mrem) par an, soit aussi les $3 / 100^{\circ}$ de la limite de $5 \mathrm{mSv}$ recommandée par la Commission des Communautés européennes (CCE) pour les personnes du public [8]. Avec les récepteurs modernes, cet équivalent de dose dépasse exceptionnellement le cinquième de cette valeur, soit $3 \mu \mathrm{Sv}(0,3 \mathrm{mrem})$ par an. Les statistiques connues actuellement font état de valeurs dans l'intervalle de 0,1 à $0,5 \mu \mathrm{Sv}$ (10 à $50 \mu \mathrm{rem}$ ) par an.

Dans le cas où les récepteurs de télévision sont utilisés comme consoles de visualisation des jeux vidéo ou des ordinateurs familiaux, la distance de vision peut descendre à $1 \mathrm{~m}$ et même en-dessous, ce qui multiplie les valeurs trouvées précédemment par 4 ou 5 . Avec les matériels modernes, ceci ne pose pas de problèmes, mais, si l'on utilise des matériels anciens, les équivalents de dose cumulés peuvent être de quelques dixièmes de millisieverts soit - quelques dizaines de millirems par an - ce qui est loin d'être négligeable bien que le niveau soit encore de $1 / 20^{\text {e }}$ de la limite recommandée pour le public. Le problème a attiré l'attention des hygiénistes américains car, aux Etats-Unis, les niveaux peuvent être plus élevés, les tubes étant alimentés sous des tensions de l'ordre de $30 \mathrm{kV}$ pour augmenter la brillance de l'écran de façon à être observé à la lumière du jour [12].

\subsection{Opérateur sur terminal à console de visualisation polychrome}

Sauf quelques cas très particuliers, les écrans cathodiques des consoles de visualisation ont la même géométrie que les écrans de télévision. Les positions de l'opérateur et de l'écran sont mieux connues et les études ergonomiques sur ces postes de travail nous ont fourni beaucoup d'informations.

Les calculs effectués partent des données de Cakir, Hart et Stewart [4]. L'agencement type du poste de travail est donné à la figure 6. Les facteurs de conversion calculés comme précédemment sont donnés dans le tableau IV. On a admis, pour ce calcul, que les gonades sont susceptibles de recevoir en permanence le rayonnement du tube cathodique parce que cette région peut être protégée ou non de ce rayonnement par la table de travail suivant la position de l'opérateur. 


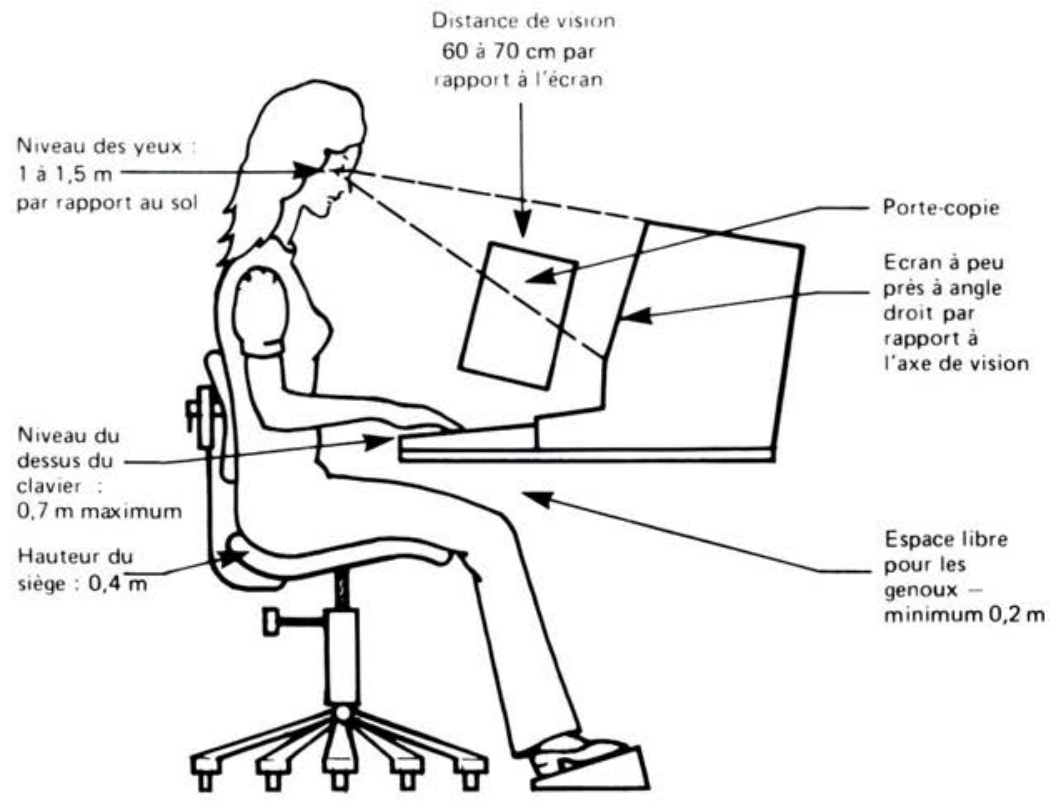

Fig. 6. - Terminal à écran cathodique. Disposition type du poste de travail pour une opératrice. Document tiré de l'ouvrage de Cakir, Hart et Stewart [4].

Dans les conditions de travail étudiées, les parties du corps les plus irradiées sont la tête et le haut du torse, et l'organe critique est le cristallin. L'horaire de travail le plus chargé est celui des opérateurs de saisie de données où l'on admet que les périodes d'activités sont de $1 \mathrm{~h}$ et demie au maximum suivie d'une pause de 1/4 d'heure. Ceci correspond à un horaire journalier de $6 \mathrm{~h}$ et annuel de $1400 \mathrm{~h}$. En procédant comme précédemment on obtient les limites d'exposition que les opérateurs sont susceptibles de recevoir avec des terminaux trichromes. Ces résultats sont rassemblés dans le tableau $V$. On remarquera que seule la valeur de $0,2 \mu \mathrm{Sv} / \mathrm{h}$ a été prise en compte pour le débit d'équivalent de dose à $5 \mathrm{~cm}$ de l'écran. Cela tient au fait que les consoles trichromes sont apparues très récemment et ont donc bénéficié des derniers perfectionnements techniques.

Ces valeurs sont au moins 250 fois plus faibles que les limites recommandées pour les personnes du public. Elles correspondent à l'observation d'images à fond blanc. Dans le cas de l'affichage de caractères lumineux sur fond noir, il faut diviser ces chiffres par un facteur de 100 à 10000 . Si l'on se base sur les statistiques récentes des récepteurs de télévision, on peut encore diviser ces valeurs par 10 . 


\section{G. WOLBER}

\section{TABLEAU IV}

Facteurs de conversion calculés pour obtenir la dose aux organes d'un opérateur sur terminal à console de visualisation équipée d'un tube polychrome alimenté à $25 \mathrm{kV}$. La distance de vision est de $60 \mathrm{~cm}$.

\begin{tabular}{|l|c|c|c|}
\hline $\begin{array}{c}\text { Organes soumis } \\
\text { au risque } \\
\text { d'irradiation }\end{array}$ & $\begin{array}{c}\text { Rapport de la dose } \\
\text { à la surface du point } \\
\text { de pénétration dans } \\
\text { le corps à la dose } \\
\text { à 5 cm de l'écran }\end{array}$ & $\begin{array}{c}\text { Rapport de la dose } \\
\text { à l'organe } \\
\text { à la dose à la surface } \\
\text { du corps }\end{array}$ & $\begin{array}{c}\text { Rapport de la dose } \\
\text { à l'organe à la dose } \\
\text { à 5 cm de l'écran }\end{array}$ \\
\hline Oeil (cristallin) & 0,254 & 0,81 & 0,206 \\
Crâne (os) & 0,254 & 0,77 & 0,196 \\
$\begin{array}{l}\text { Mœlle osseuse } \\
\text { (sternum) }\end{array}$ & 0,070 & 0,56 & 0,039 \\
Sein (femme) & 0,064 & 0,68 & 0,044 \\
Testicules & $2.10^{-4}$ & 0,56 & $1,12.10^{-4}$ \\
Ovaires & $2.10^{-3}$ & 0,02 & $4.10^{-5}$ \\
\hline
\end{tabular}

\section{TABLEAU $V$}

Limite supérieure des équivalents de dose reçus annuellement par les opérateurs sur terminal informatique à console de visualisation polychrome.

Débit de dose mesuré à $5 \mathrm{~cm}$ de l'écran : $0,2 \mu \mathrm{Sv} / \mathrm{h}(20 \mu \mathrm{rem} / \mathrm{h})$

\begin{tabular}{|l|l|}
\hline $\begin{array}{c}\text { Organe soumis au } \\
\text { risque d'irradiation }\end{array}$ & Equivalent de dose annuel \\
\hline Cristallin & $0,058 \mathrm{mSv}(5,8 \mathrm{mrem})$ \\
Crâne & $0,054 \mathrm{mSv}(5,4 \mathrm{mrem})$ \\
Mœlle osseuse & $0,011 \mathrm{mSv}(1,1 \mathrm{mrem})$ \\
Seins & $0,012 \mathrm{mSv}(1,2 \mathrm{mrem})$ \\
Testicules & $3,4.10^{-5} \mathrm{mSv}(3,4 \mu \mathrm{rem})$ \\
Ovaires & $1,12.10^{-5} \mathrm{mSv}(1,12 \mu \mathrm{rem})$ \\
\hline
\end{tabular}

\begin{tabular}{l|l}
$\begin{array}{l}\text { Equivalent de dose effectif : } \\
\text { Hommes }\end{array}$ & (CIPR 26) \\
Femmes & $0,010 \mathrm{mSv}(1 \mathrm{mrem})$ \\
& $0,011 \mathrm{mSv}(1,1 \mathrm{mrem})$
\end{tabular}

Evidemment, ces résultats ne concernent pas les applications de la bureautique comme le traitement de texte et les dispositifs d'affichage graphique polychrome à tube cathodique à pénétration. Comme indiqué précédemment, ces matériels n'ont pas de rayonnement $\mathrm{X}$ significatif. 


\section{DISCUSSION}

A la fin des années 1960, les constructeurs européens de tubes cathodiques s'étaient fixés un niveau limite de $1 \mu \mathrm{Sv} / \mathrm{h}$, équivalent à un débit d'exposition de $0,1 \mathrm{mR} / \mathrm{h}$ mesuré à $5 \mathrm{~cm}$ de toute partie accessible du téléviseur y compris la face avant de l'écran. Ils faisaient déjà mieux que les limites de $5 \mu \mathrm{Sv} / \mathrm{h}(0,5 \mathrm{mrem} / \mathrm{h})$ de la norme AFNOR conforme aux recommandations de la CIPR [7] et aux directives de la Communauté européenne [8].

La transistorisation totale des équipements de télévision a amélioré la situation dans des proportions telles qu'il faut tabler maintenant sur des débits de dose mesurés à $5 \mathrm{~cm}$ de l'écran de 0,2 à $1 \mu \mathrm{Sv} / \mathrm{h}(0,02 \mathrm{mrem}$ à $0,1 \mathrm{mrem} / \mathrm{h}$ ). Ces résultats sont rapportés par le NIOSH(1) [14] aux EtatsUnis à partir de mesures effectuées avec des chambres d'ionisation à haute sensibilité (seuil $20 \mu \mathrm{rem} / \mathrm{h}$ ) et par Wang [16] au cours d'une campagne de mesures effectuées avec des dosimètres thermoluminescents au sulfate de calcium sur des récepteurs commerciaux. Les Argentins ont publié des valeurs inférieures à $10^{-8} \mathrm{~Sv} / \mathrm{h}(1 \mu \mathrm{rem} / \mathrm{h})$ pour des mesures effectuées à $5 \mathrm{~cm}$ de l'écran avec des dosimètres thermoluminescents [13]. Ces derniers résultats ne sont pas fiables, selon les experts, car ces dosimètres sont plus sensibles aux photons de $10 \mathrm{keV}$ qu'à ceux de $500 \mathrm{keV}$, mais l'irradiation n'est plus homogène à l'intérieur du dosimètre. Ces niveaux ne seront pas dépassés chez l'utilisateur car il n'est pas possible, en exploitation normale, de faire varier la haute tension d'accélération et on fait varier la brillance de l'écran en jouant sur l'intensité du courant débité. Les équivalents de dose délivrés par les équipements à tube cathodique de télévision sont donc de l'ordre du $\mu$ Sv par an $(0,1 \mathrm{mrem} / \mathrm{an})$ et moins, de sorte que le problème de l'émission de rayons $X$ mous par ces dispositifs est définitivement résolu.

\section{CONCLUSION}

Les tubes cathodiques de télévision peuvent se classer en deux catégories. D'une part, il y a les tubes monochromes équipant les récepteurs de télévision noir et blanc et les matériels de bureautique qui ne donnent lieu à aucun rayonnement $X$ parasite. A cette catégorie se rattachent les dispositifs d'affichage graphique à tube couleur à pénétration. D'autre part, il y a les tubes trichromes proprement dit équipant les récepteurs de télévision couleur et les consoles de visualisation couleur apparues récemment sur le marché. Le rayonnement $X$ parasite émis par ces matériels n'est mesurable actuellement qu'à l'aide de techniques relevant du laboratoire de métrologie et les équivalents de dose reçus par les utilisateurs sont de l'ordre de $1 \mu \mathrm{Sv}(0,1 \mathrm{mrem})$ par an. Les valeurs de $2 \mathrm{mrem} / \mathrm{an}$ annoncées jusqu'ici ne sont plus conformes à la vérité.

(1) NIOSH : National Institute for occupational safety and health, Cincinatti, Ohio, Etats-Unis. 


\section{G. WOLBER}

\section{BIBLIOGRAPHIE}

[1] AFNOR. Appareils électroniques et appareils associés à usage domestique ou usage général analogue, reliés au réseau. Norme AFNOR NF - C 92-130, 1980.

[2] ATTIX F.H. et ROESCH W.C. Radiation Dosimetry. Vol. 1 : Fundamentals. New York: Academic press, 1968.

[3] BERTHAUD $M$. et PENOTET $H$. Le rayonnement $X$ des récepteurs de télévision. In: "Problèmes de radioprotection liés à l'émission de rayons $\mathrm{X}$ parasites par les systèmes électroniques", Toulouse, 3-6 Nov. 1970. EUR 4640, 1971, 213-229.

[4] CAKIR A., HART D.J. et STEWART T.F.M. Les terminaux à écran. Paris : Les éditions d'organisation, 1980.

[5] Commission internationale de protection radiologique (CIPR). Report of the Task group on reference man, adopted by the Commission in Oct. 1974. ICRP Publication 23. Oxford. Pergamon Press, 1975.

[6] Commission internationale de protection radiologique (CIPR). Recommandations adoptées le 17 janv. 1977. Publication CIPR 26 (Traduction de Mme A. Duchêne). Oxford: Pergamon Press, 1980.

[7] Commission internationale de protection radiologique (CIPR). Protection against ionizing radiation from external sources. Data for protection against radiation from external Sources. ICRP Publications 15 and 21. Oxford: Pergamon Press, 1978.

[8] Communautés européennes. Directive du Conseil portant modification des directives fixant les normes de base relative à la protection sanitaire de la population et des travailleurs contre les dangers résultants des rayonnements ionisants. J.O.C.E., n' L246, 17 sept. 1980.

[9] DELPLA M. et SCHAEFFER $R$. Les organes critiques et les rayons $X$ mous. In : "Problèmes de radioprotection liés à l'émission de rayons $X$ parasites par les systèmes électroniques", Toulouse, 3-6 Nov. 1970. EUR-4640, 1971, 523-544.

[10] FITZGERALD J.J., BROWNELL G.L., MAHONEY F.J. Mathematical theory of radiation dosimetry. New York: Gordon and Breach, 1967.

[11] KRAMER R. et DREXLER G. On the calculation of the effective dose equivalent. Radiat. Prot. Dos., 1982, 3, (1/2) 13-24.

[12] NASHEL D.J., KORMAN L.Y., BOWMAN J.O. Radiation hazard of video screens New England J. Med. 1982, 307, 891.

[13] NOLMANN C.E. et THOMACZ E. Study and application of properties of $\mathrm{CaF}_{2}$ : Dy dosimeters. Nucl. Instr. Meth., 1980, 275, (1) 68-70.

[14] Sous-commission d'études médicales de l'Union internationale des industries du gaz (UIIG). Rapport interne, 1981.

[15] STRACKEE L. et VAN DONGEN R. Measurement of $X$ rays at low exposure rates with thin $\mathrm{Nal}(\mathrm{TI})$ scintillation crystals. In : "Problèmes de radioprotection liés à l'émission de rayons X parasites par des systèmes électroniques", Toulouse, 3-6 Nov. 1970. EUR-4640, 1971, 433-452.

[16] WANG Y.S. Measurement of ionizing radiation from color television receivers by thermoluminescent dosimeters. Health Phys., 1975, 28, (1) 75-80. 